\title{
Cold-water immersion combined with active recovery is equally as effective as active recovery during 10 weeks of high-intensity combined strength and endurance training in men
}

\author{
Ritva S. Taipale ${ }^{1,2}$, Johanna K. Ihalainen ${ }^{2,3}$, Phillip J. Jones², Antti A. Mero², Keijo Häkkinen², \\ Heikki Kyröläinen ${ }^{2}$ \\ ${ }^{1}$ Kajaani University of Applied Sciences, Kajaani, Finland; ${ }^{2}$ Neuromuscular Research Center, Faculty of Sport and \\ Health Sciences, University of Jyväskylä, Finland; ${ }^{3}$ Department of Health Sciences, Swedish Winter Sports Research \\ Centre, Mid Sweden University, Östersund, Sweden
}

\section{Summary}

Study aim: The purpose of this study was to compare the effects of cold-water immersion (CWI) vs. active recovery performed after each individual strength and endurance training session over a 10-week period of high-intensity combined strength and endurance training.

Materials and methods: Seventeen healthy men completed 10 weeks of high-intensity combined strength and endurance training. One group ( $\mathrm{AR}, \mathrm{n}=10$ ) completed active recovery that included 15 minutes of running at $30-40 \% \mathrm{VO}_{2 \mathrm{max}}$ after every strength training session while the other group (CWI, $\mathrm{n}=7$ ) completed 5 minutes of active recovery (at the same intensity as the AR group) followed by 10 minutes of cold-water $\left(12 \pm 1^{\circ} \mathrm{C}\right)$ immersion. During CWI, the subjects were seated passively during the 10 minutes of cold-water immersion and the water level remained just below the pectoral muscles. Muscle strength and power were measured by isometric bilateral, 1 repetition maximum, leg press (ISOM LP) and countermovement jump (CMJ) height. Endurance performance was measured by a $3000 \mathrm{~m}$ running time trial. Serum testosterone, cortisol, and IGF-1 were assessed from venous blood samples.

Results: ISOM LP and CMJ increased significantly over the training period, but $3000 \mathrm{~m}$ running time increased only marginally. Serum testosterone, cortisol, and IGF-1 remained unchanged over the intervention period. No differences between the groups were observed.

Conclusions: AR and CWI were equally effective during 10 weeks of high-intensity combined strength and endurance training. Thus, physically active individuals participating in high-intensity combined strength and endurance training should use the recovery method they prefer.

Key words: Cold water immersion - Combined strength and endurance training - Recovery

\section{Introduction}

Recovery from exercise is important for reaping the benefits of repeated bouts of exercise. Recovery refers to the restoration of physiological processes of the body to their pre-fatigued state. In addition to sleep and nutrition, a number of recovery methods may be employed to facilitate recovery, such as: active recovery (AR, generally light aerobic exercise), stretching, massage, cryotherapy, nonsteroidal anti-inflammatory drugs, and compression garments. Cold-water immersion (CWI) is an easily accessible cryotherapy method that is commonly used. CWI can be performed in water or a combination of water and ice with water temperatures ranging from $5^{\circ}$ to $15^{\circ} \mathrm{C}$ and immersion time ranges from a few minutes to over an hour [5]. Several studies have reported decreases in skin, muscle, and core temperature with CWI that may aid recovery by reducing and/or delaying inflammation and the onset of muscle soreness [6], and increasing the feeling of relaxation [1]. In contrast, CWI may attenuate improvements in muscle mass and strength while blunting related muscle signaling processes for up to two days after exercise [4]. In terms of active recovery, the contraction-relaxation action 
of active muscles increases or maintains blood flow while facilitating the clearance of metabolic waste, decreasing body temperature, and balancing the autonomic nervous system to drive recovery [3].

It is well established that acute bouts of high-intensity training must be balanced with adequate and appropriate recovery to optimize training adaptations. Generally, adaptations to combined strength and endurance training include improved neuromuscular function and movement economy, as well as changes in body composition, but may also result in compromised adaptations in muscular function, particularly with higher volumes and intensities of combined training. The purpose of this study was to compare the effects of the contrasting recovery methods of CWI and AR performed after each individual strength and endurance training session over a 10 -week period of high-intensity combined strength and endurance training. We hypothesize that these contrasting methods may influence recovery and thus training adaptations.

\section{Material and methods}

Seventeen men completed the study. The inclusion criteria were as follows: healthy, non-smoking, recreationally physically active, BMI $<30 \mathrm{~kg} / \mathrm{m}^{2}$ and a recent Cooper running test $>2300 \mathrm{~m}$. Exclusion criteria and participant readiness have been described previously [2]. Ethical approval was granted by the University Ethical Committee, and the study was completed according to the most recent provisions stated in the Declaration of Helsinki.

\section{Study design}

The training intervention has also been described previously [2]. One group of men completed active recovery (AR, $\mathrm{n}=10$, age: $33 \pm 5$ years, height: $183 \pm 5 \mathrm{~cm}$, body mass: $81 \pm 9 \mathrm{~kg}$, body fat: $17 \pm 5 \%$ ) including 15 minutes of running at $30-40 \% \mathrm{VO}_{2 \max }$ after each strength training session, while the cold-water immersion (CWI, $\mathrm{n}=7,29$ \pm 4 years, $178 \pm 5 \mathrm{~cm}, 80 \pm 9 \mathrm{~kg}, 17 \pm 4 \%$ ) group completed 5 minutes of active recovery (at the same intensity as the AR group) followed by 10 minutes of cold-water immersion after each strength training session. Strength and endurance training sessions were performed on separate days. Strength training targeted primarily the lower extremities with several multi-joint movements performed both maximally and explosively. Endurance training consisted of $4 \times 4$ min running intervals and $3 \times 3 \times 100 \mathrm{~m}$ all-out sprints. The water temperature was maintained at $12 \pm 1{ }^{\circ} \mathrm{C}$ using a combination of water and ice. Subjects were seated passively during the 10 minutes of cold-water immersion and the water level remained just below the pectoral muscles.

\section{Measurements}

Muscle strength and power were measured by isometric bilateral, 1 repetition, maximum leg press (ISOM LP) and countermovement jump (CMJ) while endurance performance was measured by a $3000 \mathrm{~m}$ running time trial on a $200 \mathrm{~m}$ indoor running track. Serum testosterone (T), cortisol (C), and insulin-like growth factor 1 (IGF-1) were assessed from venous blood samples that were taken in the morning after a 10-12 hour fast between 7.00 and 8.00. The methods have been reported previously [2]. All measurements, except the $3000 \mathrm{~m}$ run, were completed at control (2-3 months before the intervention) and at pre ( 0 weeks), mid (5 weeks) and post (10 weeks) intervention.

\section{Statistics}

Statistics were performed using SPSS for Windows (IBM SPSS version 24.0; SPSS Inc., Chicago, IL). Means and standard deviations (SD) were calculated using conventional methods. A one-way analysis of variance was used to compare continuous variables between the groups at baseline and post-intervention, and comparisons within the groups were done using the paired samples t-test. A univariate general linear model covariance analysis (ANCOVA) was used to test differences between the groups, with the difference ( $\Delta$-value) as the dependent factor, group variable as the fixed factor, and baseline values as covariates. Mauchly's test was used to test the assumption of sphericity. Where this assumption was violated, Greenhouse-Geisser adjustments were used. Where significant main effects or interactions were observed, pairwise comparisons were used to identify the location of differences between measurement time points and training. All data are reported as means and standard deviations and statistical significance was set at $\mathrm{p} \leq 0.05$.

\section{Results}

The participants completed $97 \%$ of the 37 training sessions over the 10 -week combined strength and endurance training intervention. The results are presented in Table 1. A significant effect for time was observed in ISOM LP $(\mathrm{p}<0.001)$ and CMJ $(\mathrm{p}<0.001)$, but not in $3000 \mathrm{~m}$ running, T, C or IGF-1. No significant effect for group or group*time interaction was observed in any of the variables.

\section{Discussion}

A 10-week combined strength and endurance training intervention accompanied by either AR or CWI induced similar performance gains and no changes in basal 
Table 1. Isometric bilateral leg press (ISOM LP), countermovement jump (CMJ), $3000 \mathrm{~m}$ running test, testosterone, cortisol, and insulin-life growth factor 1 (IGF-1. $*$ = significant difference from control $\mathrm{p} \leq 0.05 ; * *$ significant difference from control $\mathrm{p} \leq 0.01 ; * * *=$ significant difference from control $\mathrm{p} \leq 0.001 ;+=$ significant difference from pre $\mathrm{p} \leq 0.05 ;++=$ significant difference from pre $\mathrm{p} \leq 0.01 ; \alpha=$ significant difference from mid $\mathrm{p} \leq 0.05$

\begin{tabular}{|c|c|c|c|c|}
\hline & Control & Pre & Mid & Post \\
\hline \multicolumn{5}{|c|}{ ISOM LP $[N]$} \\
\hline CWI & $4009 \pm 1001$ & $4287 \pm 765$ & $4418 \pm 817$ & $4802 \pm 786^{*, \alpha}$ \\
\hline AR & $3744 \pm 1071$ & $4134 \pm 934^{*}$ & $4318 \pm 1137 * *$ & $4657 \pm 1201^{* * *,+, \infty}$ \\
\hline \multicolumn{5}{|c|}{$\mathbf{C M J}[\mathrm{cm}]$} \\
\hline CWI & $33 \pm 4.9$ & $34 \pm 5.1$ & $35 \pm 5.1$ & $37 \pm 6.5^{* *,+, \alpha}$ \\
\hline AR & $35 \pm 4.8$ & $35 \pm 5.4$ & $38 \pm 5.4^{* * *,+}$ & $39 \pm 5.3 * * *,++$ \\
\hline \multicolumn{5}{|c|}{3000 m run $[\mathrm{s}]$} \\
\hline CWI & $751 \pm 51$ & $761 \pm 69$ & - & $729 \pm 54$ \\
\hline AR & $773 \pm 77$ & $787 \pm 79$ & - & $767 \pm 62$ \\
\hline \multicolumn{5}{|c|}{ Testosterone $\left[\mathrm{nmol} \cdot \mathrm{L}^{-1}\right]$} \\
\hline CWI & $14.1 \pm 2.7$ & $17.3 \pm 3.6$ & $16.9 \pm 3.6$ & $18.5 \pm 3.5$ \\
\hline AR & $15.3 \pm 3.5$ & $15.3 \pm 4.9$ & $16.1 \pm 3.5$ & $15.2 \pm 4.5$ \\
\hline \multicolumn{5}{|c|}{ Cortisol $\left[\mathrm{nmol} \cdot \mathrm{L}^{-1}\right]$} \\
\hline CWI & $501 \pm 163$ & $477 \pm 90$ & $502 \pm 110$ & $467 \pm 117$ \\
\hline AR & $458 \pm 112$ & $453 \pm 119$ & $419 \pm 81$ & $421 \pm 129$ \\
\hline \multicolumn{5}{|c|}{ IGF-1 $\left[\mathrm{nmol} \cdot \mathrm{L}^{-1}\right]$} \\
\hline CWI & $30.7 \pm 6.5$ & $30.7 \pm 8.9$ & $31.9 \pm 9.8$ & $32.3 \pm 7.2$ \\
\hline AR & $34.3 \pm 8.3$ & $33.9 \pm 10.1$ & $35.6 \pm 12.8$ & $34.4 \pm 9.8$ \\
\hline
\end{tabular}

hormonal concentrations in physically active men. No differences between the groups were observed in any of the measured variables. Only strength and power (ISOM LP and $\mathrm{CMJ}$ ) increased significantly in both groups while endurance performance improved only marginally. The observed increases in strength and power were expected while the lack of significant improvement in running time may indicate that the present training combined with AR or CWI is not effective in improving running capacity over relatively short distances on a flat surface. While CWI may have reduced or delayed muscle soreness or increased the feeling of relaxation $[1,6]$ and AR may have aided in clearing metabolic waste [3], AR and CWI did not cause differences in adaptations to the present training.

\section{Conclusions}

$\mathrm{AR}$ and CWI were equally effective during 10 weeks of high-intensity combined strength and endurance training. Individuals participating in high-intensity combined strength and endurance training should use the recovery method that suits their schedule and individual wants and needs.
Conflict of interest: Authors state no conflict of interest.

\section{References}

1. Ahokas E.K., Ihalainen J.K., Kyröläinen H., Mero A.A. (2019) Effects of water immersion methods on postexercise recovery of physical and mental performance. J. Strength Cond. Res., 33(6): 1488-1495. DOI: 10.1519/ JSC.0000000000003134.

2. Myllyaho M.M., Ihalainen J.K., Hackney A.C., Valtonen M., Nummela A., Vaara E., Häkkinen K., Kyröläinen H., Taipale R.S. (2018) Hormonal contraceptive use does not affect strength, endurance, or body composition adaptations to combined strength and endurance training in women. J. Strength Cond. Res., Published ahead of print.

3. Reilly T., Ekblom B. (2005) The use of recovery methods post-exercise. J. Sport Sci., 23(6): 619-627. DOI: 10.1080/02640410400021302.

4. Roberts L.A., Raastad T., Markworth J.F., Figueiredo V.C., Egner I.M., Shield A., Cameron-Smith D., Coombes J.S. Peake J.M. (2015) Post-exercise cold water 
immersion attenuates acute anabolic signalling and longterm adaptations in muscle to strength training. J. Physiol., 593(18): 4285-4301. DOI: 10.1113/JP270570.

5. Sanchez Ureña B., Barrantes Brais K., Ureña Bonilla P., Calleja González J., Ostojic S. (2015) Effect of water immersion on recovery from fatigue: a meta-analysis Eur. $J$. Hum. Mov., 34: 1-14.

6. White G.E., Wells G.D. (2013) Cold-water immersion and other forms of cryotherapy: Physiological changes potentially affecting recovery from high-intensity exercise. Extrem. Physiol. Med., 2(1): 26. DOI: 10.1186/20467648-2-26.
Received 11.09.2019
Accepted 18.11.2019

(C) University of Physical Education, Warsaw, Poland

\section{Acknowledgments}

The authors thank the Finnish Cultural Foundation and the unit of Biology of Physical Activity for financial contributions to this work. A heartfelt thank you is also extended to Biology of Physical Activity technical staff and students as well as to our study participants. 